\title{
Reversible Hepatic Cytolysis Secondary to Sunitinib in Metastatic Renal Carcinoma
}

\section{Omar EI Mesbahi and Fatima Zahra EI M'rabet*}

Department of medical oncology, University Hospital Hassan II Fez Morocco

\begin{abstract}
Liver damage is further characterized into hepatocellular (predominantly initial Alanine transferase elevation) and cholestatic (initial alkaline phosphatase rise) types. However they are not mutually exclusive and mixed type of injuries are often encountered. Serious drug-induced hepatotoxicity is an infrequent but life-threatening complication often identified through post marketing drug safety surveillance. The main toxicities of Tyrosine Kinase Inhibitors (TKI) therapy are fatigue, rash, diarrhea, hypertension, stomatitis, hand-foot syndrome, hypothyroidism and cardiac toxicity. Whilst most multitargeted TKI exhibit most of the side-effects noted above, each TKI has its particular profile. Sunitinib is an oral multikinase inhibitor that blocks the activity of VEGFR-2 and PDGFR, as well as Src, Abl, insulin-like growth factor receptor-1 and fibroblast growth factor receptor-1 tyrosine kinases, approved by the United States Food and Drug Administration (FDA) in January 2006 for treatment of renal cell carcinoma and gastrointestinal stromal tumor after disease progression or intolerance of Imatinib mesylate. In preapproval clinical trials, two patients reportedly experienced hepatotoxicity during treatment with sunitinib. Although both patients had evidence of liver metastasis before receiving sunitinib, the FDA deemed the suggestion of hepatotoxicity equivocal. We report the case of a patient treated for metastatic renal carcinoma who presented a hepatic cytolysis after introduction the sunitinib: $50 \mathrm{mg} / \mathrm{day}$, this is a second report case in the English literature in our knowledge.
\end{abstract}

Clinicians should be aware of this possible adverse effect of sunitinib, and continued pharmacovigilance is imperative to accurately quantify the possible risk of sunitinib-related hepatotoxicity.

Keywords: Hepatotoxicity; Sunitinib; Adverse effect; Tyrosine kinase inhibitors; Metastatic renal cell carcinoma

\section{Introduction}

Drug-induced hepatotoxicity is an infrequent but life-threatening complication [1]. Sunitinib is a multitargeted receptor tyrosine kinase inhibitor approved for treatment of advanced renal cell carcinoma and gastrointestinal stromal tumor [2]. The most Common treatmentemergent adverse events in the same patients during sunitinib therapy were hypertension (15-28\%), fatigue (42-74\%), headache (13-25\%), skin rash (14-38\%), hyper pigmentation (30-33\%), dry skin (17\%), hand-foot syndrome (12-14\%), diarrhea (40-55\%), nausea (31-54\%), mucositis/stomatitis (29-53\%), dyspepsia (46\%), anorexia $31-33 \%)$, neutropenia (53\%), thrombocytopenia (38\%), bleeding (18-26\%), LVEF decreased (10\%), hypothyroidism (4-7\%) [3-5]. Rare but serious adverse events were venous thromboembolism and reversible posterior leukoencephalopathy syndrome [6]. Hepatotoxicity occurred in two $(<1 \%)$ patients in clinical trials, both of whom had documented liver metastasis [7]. To our knowledge, the first case was reported in the English literature, it was a fulminant hepatic failure during sunitinib therapy, which was reversible after treatment discontinuation [8], and however, results from preapproval clinical trials suggest an equivocal hepatic risk profile for sunitinib. We describe a 46 -year-old men with renal Cell carcinoma who was presented a manageable and reversible Liver function disturbances after the first course of suninib.

\section{Case Report}

A 49 -year-old men, chronic smoking at 35 packs per year, occasional alcohol, was diagnosed with grade 3 renal cell carcinoma of the left kidney, with metastatic disease in the liver, bone and lungs. His medical history was significant for left nephrectomy for renal cell carcinoma in 2002. 5 years later, the patient presented an intermittent colic, swelling of the right hypochondrium with slimming unencrypted. The exam found a patient with WHO 1, hepatomegaly with liver arrow at $16 \mathrm{~cm}$, consistency hard, painful, with bilateral lenticular inguinal lymphadenopathy. Abdominal ultrasound + Doppler showed a large hepatic vascular injury, complement by angio-scanner has objectified the presence of secondary localizations liver, lung and right kidney. A liver biopsy (PBF) was objectived the liver metastasis of renal cell carcinoma. Staging: Thoraco-abdominal-pelvic and cerebral CT scan had objectified the presence of 5 in pleural parenchymal nodules of 2 upper and lower lobes for secondary lesions, large liver lesions: VIII, VII, VI, V and heterogeneous and hypo dense: $7 \mathrm{~cm}$ for the more voluminous (Figure1), 2 secondary tissue lesions of the right kidney, 3 and $1.5 \mathrm{~cm}$ and secondary vertebral locations: the thoracic, lumbar and sacral confirmed by bone scintigraphy. The laboratory monitoring and a cardio-vascular assessment were normal. Sunitinib therapy was begun in doses of $50 \mathrm{mg} /$ day orally; each 6-week cycle consisted of 4 weeks of sunitinib followed by 2 weeks without sunitinib. Physical examination and laboratory monitoring were performed throughout the treatment course. Four weeks after, biochemistry exam revealed liver cytolysis grade 3 of common toxicity criteria (version 3.0) [23] aspartate aminotransferase (AST) $=13.5 \mathrm{X}$ ULN, (normal range 3-35 $\mathrm{U} / \mathrm{L})$, alanine aminotransferase (ALT) $=19.5 \mathrm{X} \mathrm{ULN}(3-40 \mathrm{U} / \mathrm{L})$, total bilirubin $=0.4 \mathrm{mg} / \mathrm{dl}(0.2-1.2 \mathrm{mg} / \mathrm{dl})$, alkaline phosphatase $=50$ $\mathrm{U} / \mathrm{L}(20-125 \mathrm{U} / \mathrm{L})$, The patient's viral serology results were negative for hepatitis A antibody, hepatitis B surface antigen, hepatitis B core antibody, and hepatitis C antibody. After stopping treatment for 4

*Corresponding author: Fatima zahra el m'rabet, Department of medical oncology, University Hospital Hassan II Fez Morocco, E-mail: fazoumed@hotmail.com

Received November 03, 2010; Accepted December 09, 2010; Published December 15, 2010

Citation: El Mesbahi O, El M'rabet FZ (2011) Reversible Hepatic Cytolysis Secondary to Sunitinib in Metastatic Renal Carcinoma. J Cancer Sci Ther 3: 047049. doi:10.4172/1948-5956.1000056

Copyright: (C) $2011 \mathrm{El}$ Mesbahi O, et al. This is an open-access article distributed under the terms of the Creative Commons Attribution License, which permits unrestricted use, distribution, and reproduction in any medium, provided the original author and source are credited. 
weeks with regular monitoring, the patient's liver chemistries had returned to normal (Figure 2). The decision was of a dose reduction of sunitinib of 12, $5 \mathrm{mg}: 37.5 \mathrm{mg} /$ day for 4 weeks after the second course. After the reintroduction of sunitinib in the lower dose of $37.5 \mathrm{mg} /$ day for 18 months of treatment, Adverse events and findings included the following, according to Common Terminology Criteria for Adverse Events (version 3.0): grade 1 fatigue, arthralgia, and dry mouth during all treatment cycles; grade 3 hand-foot syndrome during cycle 2 and 3 , and grade 3 neutropenia during cycle 2, diarrhea and abdominal pain at the end of the forth cycle, the evolution was marked by a good clinical improvement and radiological improvement (Figure 1) and no

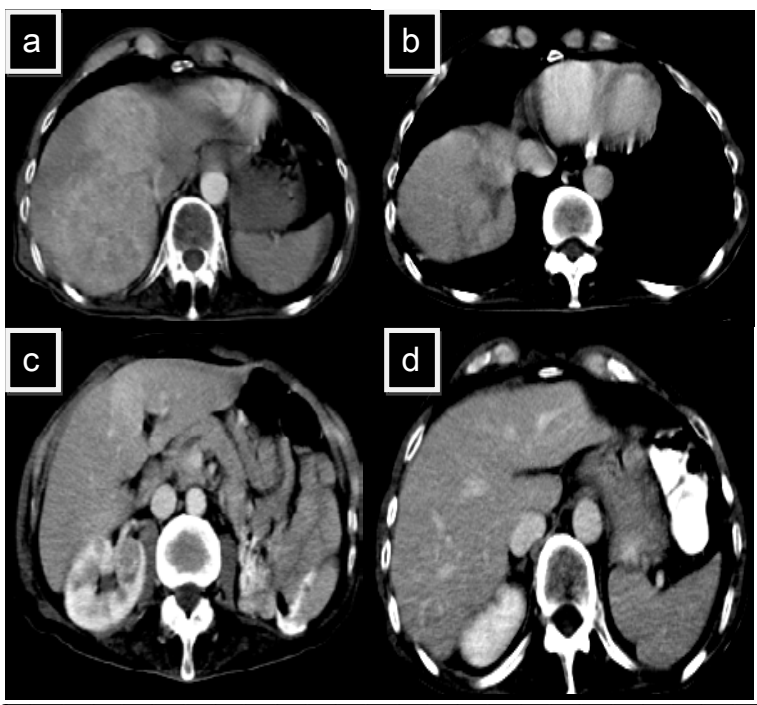

Axial CT through the liver of dynamic time objectifying the improvement of the three liver masses segments Vill (a) and V (c) enhanced significantly and became heterogeneous after
contrast. After treatment with sunitinib: decrease the size of the lesion segment Vill (b), and contrast. After treatment with sunitinib: decrod
disappearance of the lesion segment $V$ (d)
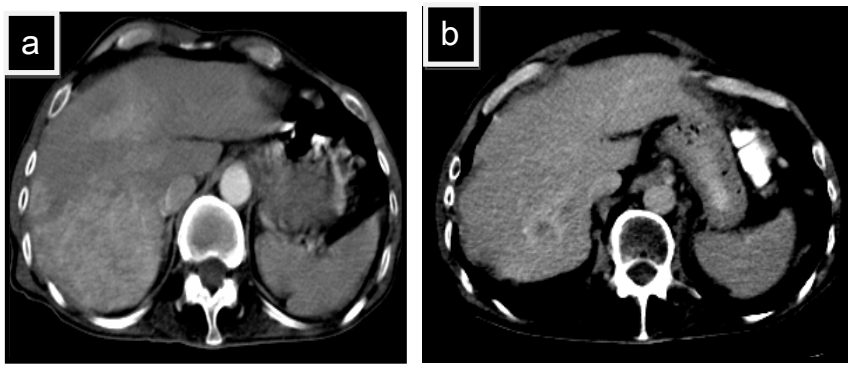

Axial CT through the liver before (a) and after treatment with sunitinib (b) objectifying the persistence of a small lesion of liver segment VII.
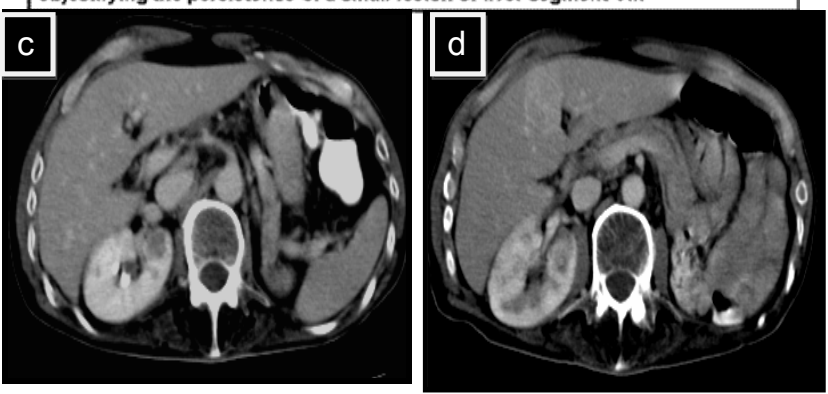

Axial CT through the right kidney objectifying the disappearance of liver lesion of the Segment $V$ (a), associated with a decrease in number and size of lesions right kidney

Figure 1: Evolution of the liver lesion after reduce dose of sunitinib.

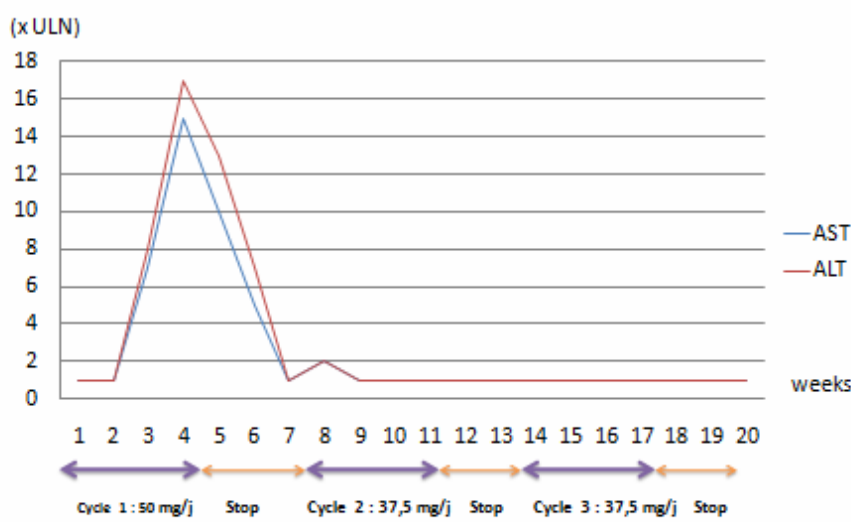

Figure 2: Evolution of the liver lesion after reduce dose of sunitinib.

deleterious side effects had been observed. The patient is currently at the $10 \mathrm{Th}$ of sunitinib treatment in partial remission.

\section{Discussion}

The treatment of metastatic renal cell carcinoma (mRCC) has recently evolved from being predominantly cytokine based to being grounded in the use of targeted agents [9]. Sunitinib, an oral multikinase inhibitor, has emerged as a front-line standard of care in Mrcc [10]. This drug is notable for its superior ORR (31\%) compared with the other agents [11]. However, sunitinib has a distinct profile of sideeffects, predominantly leukopaenia, thrombocytopaenia, stomatitis and transient skin discoloration, with skin rash and diarrhoea being less frequent, hypertension, hypothyroidism [3-5]. Cardiac toxicity had also been reported with sunitinib [4]. Hepatotoxicity occurred in two $(<1 \%)$ patients in clinical trials. Although both of whom had evidence of liver metastasis before receiving sunitinib [7]. This regoind our case report who presented a liver metastasis before treatment.

Although the exact pathophysiologies of many of these target side effects are currently undetermined, underlying risk factors may predispose some patients to certain side effects [12-13]. Each tyrosine kinase inhibitor has its particular profile. For example, stomatitis and fatigue tend to be more marked with sunitinib. Rash tends to be more marked with sorafenib [14]. Liver function disturbances tend to be more marked with pazopanib and Imatinib [15]. Imatinib mesylate was the first FDA-approved (May 2001) tyrosine kinase inhibitor [16]. This agent is the tyrosine kinase inhibitor with the most information regarding hepatotoxicity [17]. Although different from sunitinib, preclinical studies demonstrated severe Imatinib induced hepatotoxicity in dogs after 2 weeks of therapy. The frequency of imatinib-related hepatotoxicity during clinical trials was 3-6\% owing to recommendations for baseline and monthly liver function monitoring. Since FDA approval of the agent, nine additional case reports of imatinib-induced hepatotoxicity have been published [15-18]. In these reports, hepatotoxicity occurred with 2-18 months of exposure, but most often with 2-5 months. In seven patients [15-20], evidence of hepatotoxicity subsided 5-24 weeks after cessation of imatinib. One patient treated for gastrointestinal stromal tumor underwent imatinib rechallenge after resolution of hepatotoxicity; the patient immediately exhibited relapse of hepatotoxicity. Imatinib therapy was changed to sunitinib, with no evidence of recurrence after 6 months [15], two patients died, one after undergoing orthotopic liver transplantation [21]. In the English literature, the first case report of sunitinib hepatotoxicity was A 75-year-old woman who had renal cell carcinoma 
with pulmonary metastasis and was admitted to the medical intensive care unit with fulminant hepatic failure 4 days after a fifth cycle of sunitinib therapy [8]. The patient had no risk factors for alcohol-related liver disease and did not report consumption of dietary products or herbal remedies associated with hepatotoxicity. The patient's hepatic and renal chemistries had been within normal limits throughout four previous cycles of sunitinib therapy spanning 9 months. After the fifth cycle, she complained of a 3-day history of severe diarrhea and dehydration, Her abnormal laboratory test results included the Liver function disturbances. The patient's viral serology results were negative for hepatitis A antibody, hepatitis B surface antigen, hepatitis B core antibody, and hepatitis C antibody. Sunitinib therapy was stopped. After complete hemodynamic stabilization, the patient was discharged home. Laboratory tests performed at 1 and 6 months after discharge indicated that the patient's liver chemistries had returned to normal.

Our patient's 49 years old is the second case reported in the English literature in our knowledge that as risk factors for hepatic cytolysis, the history of alcoholism and presence of liver metastases before sunitinib. Contrary to what has been presented in the literature, the patient presented at the first course of sunitinib at a dose of $50 \mathrm{mg} /$ day an asymptomatic hepatic cytolysis grade 3 of common toxicity criteria (version 3.0) [23]. After stopping treatment for 4 weeks with regular monitoring, the patient's liver chemistries had returned to normal. The decision was to decrease the dose at: $37.5 \mathrm{mg} /$ day, any clinical or biochemistry deterioration was noticed. However, in the first case reported, the decision was the cessation of therapy along with supportive care, as evidenced by complete laboratory and symptom resolution 6 months after the patient's hospital discharge.

In current practice, sunitinib therapy is interrupted when patients exhibit hepatotoxicity of grade 3 or 4 . When abnormalities return to grade 1 or less, sunitinib can be reintroduced at a reduced dose.

The mechanism of sunitinib-related hepatotoxicity is unclear from the two cases reported in clinical trials [7]. One patient died of hepatic failure 11 days after sunitinib was begun; the other died 2 days after a second cycle of therapy was completed. Both patients had normal liver chemistries at baseline and during treatment. Because no histology findings were available, liver failure causality could not be determined. Given the lack of formal recommendations for hepatic monitoring during sunitinib therapy [22] and pharmacokinetic similarities with imatinib, baseline and monthly monitoring is a reasonable recommendation.

\section{Conclusion}

Although the targeted agents used in the treatment of RCC are reasonably well tolerated, their toxicity on a long term basis is unknown. As with all drugs, especially novel chemotherapeutic agents, increased reporting of adverse events in relevant cases is needed to accurately quantify the possible risk of sunitinib-related hepatotoxicity as well as other, yet unknown, toxicities. Clinicians should be aware of this possible adverse effect of sunitinib, and continued pharmacovigilance is imperative to accurately quantify the possible risk of sunitinib-related hepatotoxicity.

\section{References}

1. Navarro VJ, Senior JR (2006) Drug-related hepatotoxicity. N Engl J Med 354 731-739.
2. Faivre S, Delbaldo C, Vera K, Robert C, Lozahic S, et al. (2006) Safety pharmacokinetic, and antitumor activity of SU11248, a novel oral multitarget tyrosine kinase inhibitor, in patients with cancer. J Clin Oncol 24: 25-35.

3. Bhojani N, Jeldres C, Patard JJ, Perrotte P, Suardi N, et al. (2008) Toxicities associated with the administration of sorafenib, sunitinib, and temsirolimus and their management in patients with metastatic renal cell carcinoma. Eur Urol 53: $917-930$

4. Schmidinger M, Zielinski CC, Vogl UM, Bojic A, Bojic M, et al. (2008) Cardiac toxicity of sunitinib and sorafenib in patients with metastatic renal cell carcinoma. J Clin Oncol 26: 5204-5212.

5. Torino F, Corsello SM, Longo R, Barnabei A, Gasparini G (2009) Hypothyroidism related to tyrosine kinase inhibitors: an emerging toxic effect of targeted therapy. Nat Rev Clin Oncol 6: 219-228.

6. Vaughn C, Zhang L, Schiff D (2008) Reversible posterior leukoencephalopathy syndrome in cancer. Curr Oncol Rep 10: 86-91.

7. Center for Drug Evaluation and Research (2007) Approval package for: application Number NDA 21-938 (GIST) and NDA 21-968 (MRCC).

8. Eric W (2008) Mueller Pharmacotherapy 28: 8

9. Rini BI, Campbell SC, Escudier B (2009) Renal cell carcinoma. Lancet 373 1119-1132

10. Ravaud A, Wallerand H, Culine S, Bernhard JC, Fergelot $P$, et al. (2008) Update on the medical treatment of metastatic renal cell carcinoma. Eur Urol 54: 315325.

11. Motzer RJ, Hutson TE, Tomczak P, Michaelson MD, Bukowski RM, et al. (2009) Overall survival and updated results for sunitinib compared with interferon (IFN)-alfa in patients with metastatic renacell carcinoma (mRCC). J Clin Onco 27: 3584-3590.

12. Porta C, Szczylik C (2009) Tolerability of first-line therapy for metastatic renal cell carcinoma. Cancer Treat Rev 35: 297-307.

13. Kamba T, McDonald DM (2007) Mechanisms of adverse effects of anti-VEGF therapy for cancer. Br J Cancer 96: 1788-1795.

14. Tsai KY, Yang CH, Kuo TT, Hong HS, Chang JW (2006) Hand-foot syndrome and seborrheic dermatitis-like rash induced by sunitinib in a patient with advanced renal cell carcinoma. J Clin Oncol 24: 5786-5788

15. Pariente A, Etcharry F, Cales V, Laborde Y, Ferrari S, et al. (2006) Imatinib mesylate-induced acute hepatitis in a patient treated for gastrointestinal stromal tumour. Eur J Gastroenterol Hepatol 18: 785-787.

16. Novartis Pharmaceuticals (2007) Gleevec (imatinib mesylate) package insert November 24

17. Mindikoglu AL, Regev A, Bejarano PA, Martinez EJ, Jeffers LJ, et al. (2007) Imainib mesylate (Gleevec) hepatotoxicity. Dig Dis Sci 52: 598-601.

18. Ohyashiki K, Kuriyama Y, Nakajima A, Tauchi T, Ito Y, et al. (2002) Imatinib mesylate-induced hepatotoxicity in chronic myeloid leukaemia demonstrated focal necrosis resembling acute viral hepatitis. Leukaemia 16: 2160-2161.

19. Kikuchi S, Muroi K, Takahashi S, Kawano-Yamamoto C, Takatoku M, et al. (2004) Severe hepatitis and complete molecular response caused by imatinib mesylate: possible association of its concentration with clinical outcomes. Leuk Lymphoma 45: 2349-2351.

20. Ayoub WS, Geller SA, Tran T, Martin P, Vierling JM, et al. (2005) Imatinib (Gleevec)-induced hepatotoxicity. J Clin Gastroenterol 39: 75-77.

21. Cross TJ, Bagot C, Portmann B, Wendon J, Gillett D (2006) Imatinib mesylate as a cause of acute liver failure. Am J Hematol 81: 189-192.

22. Houk BE, Bello CL, Michaelson MD, Bukowski RM, Redman BG, et al. (2007) Exposure-response of sunitinib in metastatic renal cell carcinoma (mRCC): $A$ population pharmacokinetic/ pharmacodynamic (PKPD) approach. J Clin Oncol 25: 241s.

23. National Cancer Institute (2007) U.S. National Institutes of Health Common terminology criteria for adverse events v3.0 (CTCAE). 